\title{
Clostridium Difficile infection and Antibiotic -Associated Colitis
}

\author{
Murtaza Mustafa ${ }^{1}$, Muhammad Iftikhar $^{2}$, Saima Shafi $^{3}$, Malik J.Shah ${ }^{4}$ \\ ${ }^{1,2,4}$ Faculty of Medicine\&,Health Sciences, University Malaysia Sabah,KotaKinabalu,Sabah,Malaysia \\ ${ }^{3}$, Hospital Queen Elizabeth, Kota Kinabalu, Sabah, Malaysia
}

\begin{abstract}
$\overline{\text { ABSTRACT:Hospitalized patients receiving } \beta \text { lactam antibiotics develop diarrhea and higher rates reported }}$ in those receiving clindamycin. Clostridiumdifficile is recognized as a cause of antibiotic associated diarrhea, colitis and pseudomembranous colitis. Contributory factors include: advance age, severity of underlying illness.C.difficile has the ability to temporarily colonize newborn humans and calves, suggests that the gastrointestinal tracts of young animals may be a major reservoir.C.difficile is recognized enteric pathogen in animals including companion animals and food animals. Clinical features include: asymptomatic carriage in neonates to a fulminant, relapsing, and occasionally fatal colitis. Initial therapy for pseudomembranous colitis includes discontinuation of offending antibiotic regimen, fluid replacement and electrolyte losses. Mild to moderate infection with metronidazole, severe to recurrent cases with vancomycinTherapy with probiotic such as Sacchromyces boularadii is beneficial. Antibiotic misuse in humans and production animals must be addressed.
\end{abstract}

KEY WORDS: Clostridium difficile, Antibiotic, Colitis, and Diarrhea

\section{INTRODUCTION}

Antibiotic associated diarrhea and colitis is most common complications of antibiotic therapy.Attack rates vary depending on the antimicrobial agent used, the epidemiologic setting, and the host. Overall attack rates antibiotic-associated diarrhea in hospitals rage from 3.2\% to $29 \%$ [1].Almost $15 \%$ of hospitalized patients receiving $\beta$-lactam antibiotics develop diarrhea and rates for those receiving clindamycin rage from $10 \%$ to $20 \%$ [2].Clostridium difficile is now recognized as a frequent cause of antibiotic associated diarrhea and colitis, and the incidence of C.difficile associated diarrhea seems to be increasing [3].It is implicated in $20 \%$ to $30 \%$ of patients with antibiotic-associated diarrhea, in $50 \%$ to $75 \%$ of those with antibiotic- associated colitis [4].Literature from the mid-1970s emphasized that attack rates for diarrhea and pseudomembraneous colitis associated with the use of individual antibiotics. Several studies rates of clindamycin and lincomycin associated diarrhea ranging from $7 \%$ to $21 \%$ and rates of ampicillin-associated diarrhea from $4 \%$ to17\% [5].In the late 1970s,a series of investigations established toxigenic C.difficile as the cause of pseudomembranous colitis [6].The reported incidence of ofC.difficile in acute-care hospital increased from 30 to 40 cases per 100,000 population in 1990s to 84 per 100,000 by 2005 [7]. Although colonization with toxigenic C.diffiile occurs frequently among residents of some extended-care and rehabilitation facilities symptomatic disease develops in only a minority of infected patients [8].Animal studies found that intracecal materials transferred the disease from affected hamsters to healthy ones that both the cultures of Clostridia and their cell-free supernatants produced this disease and this activity was neutralized by gas gangrene antiserum [9].Ritkin and associates reported that stool filtrates from the humans with pseudomembranous colitis were lethal for hamsters; caused edema,hemorrhage, and increased vascular permeability in rabbit skin and possessed cytotoxic activity that was neutralized by Clostridium sordelli antitoxin [10]. Emergence of strains causing C.difficile infection in the community (CA-CDI) is becoming more commonplace [11].Unlike health care infections, CA-CDI is associated with younger, healthy people, often without prior exposure to antimicrobials or contact with hospitalized patients [12].The rates of human infection CDI have increased dramatically and C.difficile is recovered from Australian production animals $[13,14]$.The paper reviews pathophysiology, clinical features, diagnosis and therapy of antibiotic associated colitis.

\section{PATHOPHYSIOLOGY}

C.difficle most often causes a nonspecific colitis.However, especially in more severe cases, one may see the distinct macroscopic appearances of pseudomembranous colitis. The colonic mucosa is studded with adherent,raised,white and yellowish plaques.Initially,these lesions are small and discrete and are easily dislodged; the intervening mucosa may be inflamed and covered with mucus, but often it appears entirely normal [15].With progression of disease, pseudomembranous plaques may enlarge and coalesce. Pseudomembranes can exist throughout the entire colon, but they are usually most pronounced in the recto sigmoid 
colon; rarely does the disease progress proximal to the ileocecal valve [15].Histological criteria for pseudomembranous colitis and method for grading lesions have been described. The principal features are inflamed mucosa with a neutrophil predominance and mucin distended glands. Attached pseudomembranous are composed of a loose network of mucin, neutrophils, fibrin, and nuclear debris [15].

\section{RESERVIORS OF INFECTION}

Researchers have documented the prevalence of C.diffficile (or one of its toxins) in $15 \%$ to $70 \%$ of neonates [16].Despite the presence of toxin-producing organisms in this population, the prevalence of $C$.difficile colitis remain low [17].Enhancement of chemotactic responses of granulocytes to toxin $\mathrm{A}$ in older persons and the absence of high-affinity receptors for toxin A in neonates (in a rabbit model) have been cited as possible reasons for this age-dependent susceptibility $[18,19]$. The ability of $C$.difficile to temporarily colonize newborn humans, hamsters, and calves suggests that the gastrointestinal tract of very young mammals may be a major reservoir [19,20].In healthy adults, intestinal carriage rates of toxigenic C.difficle are typically 3\% or less and not greater than $8 \%$.Asymptomatic intestinal carriage rates are higher (-approximately 20\%) among hospitalized adults, particularly those who have received antibiotics [21,22].

C.difficile is a recognized enteric pathogen in a variety of animals including companion animals e.g. Cats dogs,horses, and food animals (cattle,sheep,goats,pigs)[23].In Australia C.difficile has been isolated from piglets,sheep,lambs,horses,cats,digs and cattle with the highest prevalence in neonatal animals due to a lack of established gut flora at birth. For this reason predisposing antibiotics may not be required for development of $\mathrm{CDI}$ in young animals although there is anecdotal evidence in Australia of routine use of extended-spectrum cephalosporins in production animals. This is particularly concerning in the pork industry where gross contamination of facilities with C.difficile spores is commonplace with C.difficile [24]. Several meat products, seafood, ready-to eat salad leaves and vegetables are also contaminated with C.difficile, predominantly ribotype 078-like strains [16].Contamination may occur through spillage of gut contents at slaughter or direct contamination by food handlers during dressing or retailing. Environmental contamination may also play a role. C.difficile spores survive in treated piggery effluent, the byproducts of which are often applied to agriculture land, used in retail compost manufacture, or recycled within swine facility [25].

Outside Australia, the increasing prevalence of PCR ribotype 078 in humans, food production animals and food products suggest potential zoonotic transmission. In Netherlands, where infections with ribotype 078 increased more than fourfold from 2005 to 2008.patients infected with this ribotype were younger and acquired C.difficile in the community more frequently particularly if they lived in rural pig producing areas [26]. In the USA,the prevalence of ribotype 078 infections in humans increased from $0.02 \%$ to $1.3 \%$ (pre 2001 to 2006) and ribotype 078 is increasingly associated with CA-CDI.These strains are indistinguishable or very closely related to animal ribotype 078 strains by PFGE analysis [27].Ribotype 078 strains from Dutch humans and pigs are indistinguishable by MIVA subtyping [28].Transmission from humans to animals may occur.C.difficile can be isolated from the feces of hospital pet therapy dogs that had prior negative culture for C.difficile.These dogs were > 2 times likely to be colonized with C.difficile than dog's not vising hospital [29].

\section{EXASPERATE AGENTS}

Almost all antibiotic classes have been associated with the disease, reports of large clinical series' most commonly implicate clindamycin,penicillins,cephalosprins and more recently fluroquinolones [30].Several noteworthy studies primarily implicated clindamycin and ampicillin in the 1970s [31].In the most recent outbreaks associated with NAPI/027 (North American pulsed field type 1, ribotype027) which is resistant to fluoroquinolones,fluoroquinolones are clearly identified as a risk factor for clinical disease [32].Third generation cephalosporin's have been implicated in particular and seem to predispose to C.difficile-associated disease more commonly than do the narrow spectrum penicillin's or $\beta$-lactam-stable penicillin [33].A number of antineoplastic agents particularly those with modest antibacterial activity,have been associated with C.difficile diarrheal disease, including doxorubicin, cisplatin,cyclophosphamide and others [34 ].

\section{CONTRIBUTORY FACTORS}

Antimicrobials use is the primary factor in the disease, but certain host and environmental factors also predispose to C.difficile-associated disease. In a comprehensive prospective study of risk factors in hospitalized patients, McFarland and associates[35], identified advance age and severity of underlying illness as factors associated with increased risk of C.difficile carriage and diarrhea and found that agents that alter normal intestinal motility-specifically enemas and gastrointestinal stimulants, as well as stool softener-also contribute to the risk of C.difficle associated diarrhea [35].Given the tendency of this disease to affect ill elderly patients who receive antibiotics, it is no surprise that patients with C.difficile are also at risk of colonization by vancomycin resistant enterococci [36].Other investigators have reported that critically ill burn patients, uremic patients, patients with hematologic malignancies, and those undergoing gastrointestinal surgery are at high risk of 
C.diffiile diarrhea and colitis [37-40].Emerging evidence suggests that immunologic susceptibility may play a critical role in C.difficile-associated infection. Host immunoglobulin G responses have been shown to protect against symptomatic disease and relapse [41,42].Human deficiency virus (HIV) infection per se does not appear to predispose to C.difficile colonization and specific risk factors for C.difficile- associated disease among HIVinfected patients appear to be similar to those HIV seronegative persons [43,44].In recent years CA-CDI has become more common, and in nearly one fourth of such cases, no traditional risk factor are identified [45]

\section{CLINICAL FEATURES}

Infection with toxigenic C.difficile causes a spectrum of disease ranging from asymptomatic carriage (particularly in neonates) to a fulminant, relapsing, and occasionally fatal colitis. When C.difficile produces clinical disease, the onset of signs and symptoms typically occurs after 3 to 5 days of antibacterial treatment, but diarrhea may develop as early as first of therapy or as late as 10 weeks after cessation of therapy [46].C.difficile diarrhea may be brief and self-limited or it may be cholera like, resulting in more than 20 stools per day [2].Accompanying findings often include fever (30\% to 50\% of patients), leukocytosis (50\% to 60\%) and abdominal pain or cramping (20\% to $33 \%$ ) [47,48,47].The mean peripheral leukocyte count of patients with C.difficile-associated diarrhea typically 15,000 to $16,000 / \mathrm{mm}^{3}$ in one series.C.difficle infection was noted in $25 \%$ of patients with white cell counts of greater than of greater than $35,000 / \mathrm{mm}^{3}$ who did not have hematologic malignancy [49].Nausea, malaise,anorexia, hypoalbuminemia,occult colonic bleeding, and dehydration have also been reported [2].Infrequently, $C$.difficile colitis manifests without diarrhea as an acute abdominal syndrome or toxic megacolon [50].In one report,5 of 97 patients with C.difficile disease(only 1 of whom had profuse diarrhea) presented initially with marked leukocytosis (white cell count $>25,000 / \mathrm{mm}^{3}$ ) and right lower quadrant peritoneal signs mimicking those of acute peritonitis [51].Toxicmegacolon is suggested by acute dilatation of colon to a diameter greater than $6 \mathrm{~cm}$, associated systemic toxicity, and the absence of mechanical obstruction. It carries a high mortality rate (64\% in one series of 11 patients) [52].Other intra-abdominal complications include colonic perforation, transverse volvulus, protein-losing enteropathy, and recurrent C.difficile-associated diarrhea, the last occurring in approximately $20 \%$ of patients $[53,54,55]$.Extra intestinal manifestation occur more rarely and include bacteremia, often with concurrent isolation of other constituent organisms in the bowl flora, splenic abscess, and osteomyelitis[56,57,58].In addition, multiple reports have described reactive arthritis or tenosynovitis or both $[59,60]$.

Consideration in the differential diagnosis of antibiotic-associated colitis include diarrhea caused by other enteric pathogens [especiallySalmonella), adverse reactions to other medications, ischemic colitis, inflammatory bowel disease, and intra-abdominal sepsis [50].

\section{DIAGNOSTIC METHODS}

C.difficile-associated disease should be suspected in patients with diarrhea who have received antibiotics within the past two months or whose diarrhea begins 72 hours after hospitalization [50].In most instances, toxin testing or C.difficile culture of a single stool specimen effectively establishes the diagnosis; however, repeat testing or endoscopy, or both may be necessary

\section{C.difficile toxin detection}

The most widely used means of diagnosing C.difficile-associated diarrhea and colitis clinically detection of C.difficile toxin in stool specimens. The cytotoxicity assay has been considered the gold standard for diagnosis, but most clinical laboratories use various enzyme immunoassays, which perform reasonably well compared with cytotoxicity assays [6].Stool filtrate are incubated with mammalian tissue culture cell line with and without toxin-neutralizing antibody. If toxicity activity, usually manifested as surrounding of cultured cells, is noted in the well with stool filtrate and toxin-neutralizing antibody, the test for the specimen is considered to be positive [6].If used in appropriate clinical setting, this test is both sensitive and specific; more than $90 \%$ of patients with pseudomembranous colitis have cytotoxicity in their stool detected by this assay [61].

C.difficile toxin A or B can be detected by enzyme-linked immunobsorbent assay kits, available commercial kits, their performance characteristics have been studied extensively [62].In general, they are rapid, relatively inexpensive, and specific, but lack the sensitivity of the technically more cumbersome cytotoxin assays.If measured against strictest diagnostic criteria that include clinical diarrhea, along with cytotoxin assay and culture results, their sensitivity rages from $63 \%$ to $94 \%$ with a specificity of $75 \%$ to $100 \%$ [63].

\section{C.difficledetection by culture}

Anaerobic culture for C.difficile, the most sensitive test in many laboratories, remains essential for epidemiologic studies of outbreaks.However, cost and convenience issues have driven many hospital laboratories to replace routine cultures with more rapid and less expensive enzyme-linked immunosorbent assay based assays, sometimes in combination with cytotoxinassays. Most clinical microbiology laboratories are not 
equipped to distinguish between nonpathogenic, on toxigenic strains, and testing schemes that rely solely om C.difficile cultures yield a significant number of false-positive results (In some hospitals, $20 \%$ to $25 \%$ of C.difficie isolates are non-toxigenic [64].Testing for stool toxin simultaneously or using in vitro toxin production assays my help to resolve this problem [63].

\section{Endoscopy for detection of pseudomembrane}

The detection of exudative plaques or pseudo membranes on colonic mucosa establishes the diagnosis of pseudomembranous colitis. The pathognomonic lesions is characteristically raised, yellowish, and usually 2 to $10 \mathrm{~mm}$ in diameter with "skip" of normal mucosa, but in severe disease, lesions may coalesce to form plaques [50].At least $90 \%$ of patients with pseudomembranous colitis demonstrate either C.difficile or its toxin in stool samples [48].Because of its cost, risk to the patient, and availability of other diagnostic tests, endoscopy is usually reserved for special situations [50].

\section{THERAPY}

Treatment of choice in Clostridium difficile-associated diarrhea and colitis for adults include: Initial therapy for pseudomembranous colitis should commence with discontinuation of the offending antibiotics regimen, if possible, and replacement of fluid and electrolyte losses. In a prospective treatment trial the diarrhea resolved before initiation of therapy in $23 \%$ of 149 patients, but most patients require specific antibacterial therapy [65].Therapy of Clostridium difficile-associated diarrhea and colitis for adults begins with replace fluid and electrolytes, if clinical situations allows, discontinue offending antibiotic, and avoid antiperistaltic agents plus for mild to moderate infection metronidazole $500 \mathrm{mg}$ orally 3 times daily for 10 to 14 days.In severe infection or unresponsiveness to or intolerance to metronidazole, vancomycin $125 \mathrm{mg}$ daily 4 times daily for 10 to 14 days. For second recurrence,vancomycin in tapered and pulsed doses a) $125 \mathrm{mg} 4$ times daily for 14 days, b) $125 \mathrm{mg}$ orally twice daily for 7 days, c) $125 \mathrm{mg}$ once daily for 7 days, d) $125 \mathrm{mg}$ orally once every 2 days for 8 days) $125 \mathrm{mg}$ once every 3 days for 15 days [15].Other options for recurrent infection include:Vancomycin followed by rifamiximin, $400 \mathrm{mg}$ orally 2 times daily for 14 days. Therapy with probiotic such as Sacchromyces boulradii [15].

Antiperistaltic agents, such as loperamide and diphenoxylate hydrochloride with atropine should be avoided. There is little evidence that such agents lead to symptomatic improvement, and in one study predating the discovery of Clostridumdifficile as a pathogen, diarrhea was actually more common among those receiving diphenoxylate-atropine plus lincomycin than those receiving placebo plus lincomycin [66].Several ancedotes and case series have associated the use of diphenoxylate and of lapromide and other anti-peristaltic agents with the development of toxic mega colon in patients with C.difficiledisease or pseudomembranous colitis [51].

\section{CONCLUSION}

Diarrhea and colitis is most common complications of antibiotic therapy.C.difficle causes diarrhea in humans and animals. Toxigenic C.difficleis also cause of pseudomembranous colitis.CA-CDI strains causing infection in the community is also common.Antimicrobial use in humans and animals must be addressed.

\section{REFERENCES}

[1] McFarland IM,SurawiczCM,GreenbergRN,etal..prevention of beta-lactam-associated diarrhea by Sacchromycesboulradii compared with placebo .Am JGastroenterology. 1995;90:439-448.

[2] Bartlett JG, Antibiotic-associateddiarrhea.Clin Infect Dis.1992;15:573-81.

[3] DailalRM,HarbrechtBG,BoujoukasAJ,et al. Fulminant Clostridium difficle:an underappreciated and increasing cause of death and complications Am Surg2002;235:363-72.

[4] KelleyCR,Pothoulokis C, LaMont IT .Clostridium difficlecolitis.N.Engl J Med.1994; 350:257-62.

[5] LaskRH,Fekety FR Jr, Silva Jr, et al.Gastrointestinal side effects of clindamycin and ampicillin therapy.J Infect Dis.1977;135(suppl):5111-5119.

[6] Larson HE, Parry JV, Price AB ,et al. Undescribed toxin in pseudomembranous colitis.Br Med J.1977;1:1246-48

[7] KelletCR,LaMont JT. Clostridium difficle-more difficult than everEngl JMed.2008;359:1932-40.

[8] Johnson S,HomannSR,BettinKM,etal.Treatment of asymptomatic Clostridium difficle carriers(fecal excretor) with vancomycin or metronidazole a randomized plcebo-controlled trial.Ann Intern Med.1992;117:297-302.

[9] BarlettJG,OnderdonkAR,CisnerosRI,et al Clindamycin associated colitis due to a toxin- producing species of Clostridium in hamsters.J Infect Dis.1977;136:701-705

[10] Levine I,DykoskiRK,Jrof EN. Candida - associated diarrhea: a syndrome in search of credibility.Clin Infect Dis.1995;21:581-86.

[11] Khanna S,etal.The epidemiology of community -acquired Clostridium difficle infection: a population based study. Am J Gastroenterology.2012;107:89-95

[12] NaggieS, etal.Community-associated Clostridium difficle infection: experience of a veteran affairs medical centre in southern USA.Infection.2010;38:297-300.

[13] WestrenAustralia,Department of Health,PublicHealth.Healthcare Infection Surveillance:Westren Australia Quarterly Aggregate Report,8.http://www.health. Wa.govau/cproot/4496/2/q1-2012.agg-report.pdf. 
[14] Knight DR,etal.Prevalence of Clostridium difficle carriage in Australian cattle of various ages.2012. (Submitted for publication)

[15] ThielmanNM,WilsonKH,Antibiotic -Associated Colitis. In Mandell,DouglasBennett's Principles and Practice of Infectious Diseases,7thed .Mandel GI.,BennetteJE,Dolin R (editors).Churchill Livingstone Elsvier,2010.

[16] Bolton RP,TailtSK,DearPR,et al.Asymptomatic neonatal colonization by Clostridium difficle.Arch Dis Child.1984;59:477-72

[17] el-MollandesAF,KeiserJF,RefatM,etal.Prevalence and toxigenicity of Clostridium difficle isolates in fecal microflora of preterm infants in the intensive care nursery .BiolNeonate.1993;63:225-29.

[18] Triadafilopolous G, Shah MH,PothoulakisC.Thechemostatic response of human granulocytes to Clostridium difficle toxin A is age dependent.AM JGastroenterology.1991;86:1461-65.

[19] EglowR,PothoulakisC,ItzkowitzS,et al.Diminished Clostridium difficle toxin A sensitivity in newborn rabbit ileum is associated with decreased toxin A receptor. J Clin Invest.1992;90:822-29.

[20] Rolle R,Iaconis JP. . Intestinal colonization of infant hamsters with Clostridium difficle.InfectImmune.!983;42:480-86.

[21] Nakamura S,MikavaM,NakshinS, et al.Isolation of Clostridium difficle from the feces and antibody in sera of young and elderly adults.Microbiol Immunol.1981;25:345-51

[22] Asha NJ,Wilcox MH. Laboratory diagnosis of Clostridium prefringensantibiotic-associated diarrhea Med Microbiol.2007;51:891894

[23] RuprikM,SongerJG.Clostridiumdifficle: Its potentials as a source of food borne diasese.Ad Food Nutr Res.2010;60C53-66.

[24] HopmanNE,et al. .Acquisition of Clostridium difficle by piglets. VetMicrobiol .2011;149;186-9

[25] Squire MM,etal.Detection of Clostridium difficle after treatment in a two- stage pond system In Manipulating Pig Production(van Barneveld.RJ.,ed),pp.215,Australasian Pig Science-Association.2011.

[26] GoorhuisA,et al.Emergence of Clostridium difficle infection due to a new hypervirulent strain ,polymerase chain reaction ribotype 078.Clin InfectDis.2008;47:1162-70

[27] JhungMA ,etal.Toxinotype V Clostridium diffficle in humans and food animals. Emerg Infect Dis.2008;14:1039-45.

[28] DebastSB,etal.Clostridiumdifficle PCR ribotype 078 serotype V found in diarrheal pigs identical to isolates from affected humans. Environ Microbiol.2009;11:505-11.

[29] Lefebvre SI, Weese JS .Contamination of pet therapy dogs with MRSA and Clostridium diffficle.JHosp Infect.2009;72:268-69.

[30] Robertson MB,BreenKJ,DesmondPN,etal.Incidence of antibiotic-related diarrhea and pseudomembranous colitis a prospectice study of lincomycin,clindamycin and ampicillin.Med J Aust.1977;1:243-46.

[31] LaskRH,Fekety FR Jr,Silva J Jr,et al.Gastrointestinal side effect of clindamycin and ampicillin therapy Infect Dis.1977;135(suppl):S111-S19.

[32] Loo VG, Poirer L, Miller MA, et al. A predominantly clonal multi-institutional outbreak of Clostridium difficile-associated diarrhea with high morbidity and mortality. N Eng. J Med. 2005;353:2442-2449.

[33] AnandA,Bashey B, Mir T,etal.Epidemiology,clinical manifestations and outcome of Clostridium diffficle-associated diarrhea.Am J Gastroenterol.1994;89:519-25

[34] AnadA,GlatGE.Clostridiumdifficle infection associated with antineoplastic chemotherapy: a review .Clin Infect Dis.1993;17:10913.

[35] McFarland IV,SurawiczCM,StammWE.Risk factors for Clostridium diffficle carriage and C.diffficle associated diarrhea in a cohort of hospitalized patients J InfectDis.1990;162:678-684.

[36] Ray AJ,DonskeyCI.Clostridiumdifficle infection and concurrent vancomycin resistant Enterococcus stool colonization in a health care worker: case report and review of the literature. Am J Infect Control.2003;31:54-56.

[37] GrubeBJ,HeimhaciaDM,MarvinJA.Clostridiumdifficle diarrhea in critically ill burned patients. Arch Surg.1987;122:655-61

[38] AronssonB,BaranyP,NordCE,etal.Clostridiumdifficle associated diarrhea in uremic patients. Ear J Clin Microbiol.1987;6:352-56.

[39] Heard SR,Wren B, BernnettMJ,et al Clostridium difficileinfection in patients withhaematological malignant disease risk factors,faecal toxin andpathogenic strains.Epidemiol Infect.1988;100:63-72.

[40] KeighleyMR,BurdonDW,Alexander Williams J,et al.Diarrhea and pseudomembranous colitis after gastrointestinal operations: a prospective study. Lancet.1978;2:1165-67

[41] KyneI,WarnyM,QamarA,etal.Asymptomatic carriage of Clostridium difficle and serum levels of IgG antibody against toxin A.N Engl J Med.2000;342:390-97

[42] Kyne I, WarnyM,QamarA,etal.Association between antibody response to toxin A and protection against Clostridium difficle diarrhea.Lancet.2001;357:189-93.

[43] HutinY,CasinI,LespritR,etal.Prevalence and risk factors for Clostridium diffficile colonization at admission to infectious diseases ward.Clin Infect Dis.1997;24:920-24

[44] HutinY,MolinsJM,CasinI,etal.Risk factors for Clostridium diffficle associated diarrhea in HIV-infected patients.AIDS.1993;7:144147.

[45] Surveillance for community -associated Clostridium diffficle Conneticut.2006-MMWRMorb Mortal Weekly Rep.2008;57:340-43.

[46] Tedesco FJ.Pseudomembranouscolitis.MedClin North Am.1982;66:655-64.

[47] GerdingDN,OlsonMM,PetersonIR,etal.Clostridiumdiffficle associated diarrhea and colitis in adults: a prospective case-controlled epidemiologic study. Arch InternMed.1986;146:95-100.

[48] GobhardRI,GendingDN,OlsonMM,et al. Clinical and endoscopic findings in patients early in the course of Clostridium diffficleassociated pseudomembranous colitis. AmJ Med.1985;78:45-48

[49] BartletJG,GorbochSI.Pseudomembranousenterocolitis(antibiotic-related colitis)AdvIntern Med.1977;22:455-76.

[50] FebetyR.Guidelines for the diagnosis and management of Clostridium diffficle associated diarrhea and colitis. American College of Gastroenterology; Practice Parameters Committee.Am J Gastroenterol.1997;92:739-50.

[51] DrapkinMS,Worhington MG, Chang TW,et al.Clostridium difficle mimicking acute peritonitis. Arch Surg.1985;120:1321-22

[52] TrodelJI,DeschenesM,MayandS, etal.Toxicmegacolon complicating pseudomembranous enterocolitis.Dis Colon Rectum.1995;38:1033-38. 
[53] SnooksSJ,HughesA,Horsburgh AG ,Perforated colon complicating pseudomembranous colitis.Br J Surg.1984;71:291-92.

[54] YaseenZH,WatsonRE,DeanHN,et al. Case report colon volvulus in a patient with Clostridiumdifficle pseudomembranous colitis .Am J Med Sci.1994;308:247-

[55] Do AN,FridkinSK,YechouronA,etal.Risk factors for early recurrent Clostridium diffficle associated diarrhea.Clin Infect Dis.1998;26:954-59.

[56] 56. Feldman RJ, Kallich M, Weinstein MP. Bacteremia due to Clostridium difficile: case report and review of extraintestinal C. difficile infections. Clin Infect Dis. 1995;20:1560-1562.

[57] ByiB,Jacobs F, StruedensMJ,et al Experimental Clostridium difficle infections .ClinInfect Dis.1996;22:712.

[58] SaginurR,FogelR,BeginI,et al. Splenic abscess due to Clostridium difficle.J InfectDis.1983;147:1105

[59] Atkinson MH,MclendBD,Reactive arthritis associated with Clostridium diffficle enteritis Rheumatol.1988;15:520-22

[60] Lofgren RP,TadlockIM,Soltis RD. Acute oligoarthritis associated with Clostridiumdiffficle pseudomembranous colitis. Arch Intern Med.1984;144:617-19.

[61] Lyerty DM ,KrivanHC,WikinsTD.Clostridiumdiffficle: its disease and toxins.ClinMicrobiol Rev.1988;1:18

[62] GroschelDH.Clostridiumdiffficleinfection.Clin Infect Lab Sci.1996;33:203-15.

[63] GerdlingDNJohnson S, Peterson IR,etal.Clostridiumdiffficle associated diarrhea and colitis. Infect Control Hosp Epidemiol.1995; 16:459-477

[64] Cohen SH, Tang YJ, Muenzer J, et al. Isolation of various genotypes of Clostridium defficile from patients and the environment in an oncology ward. Clin Infect Dis. 1997;24:889-893.

[65] Teasley DG, Gerding DN, Olson MM,et al. Prospective randomized trial of metronidazole versus vancomycin for Clostridium difficle associated diarrhea and colitis.Lancet.1983;2:1043-46.

[66] Novak E,LeeJG,SekmanCE,et al. Unfavorable effect of atropine diphenoxylate (Lomotil) therapy in lincomycin caused diarrhea.JAMA.1976;235:1451-54. 